\title{
PROJECT SCHEDULING OPTIMIZATION IN ELECTRICAL POWER UTILITIES
}

\author{
Cleber Mira $^{1 *}$, Paulo R. Viadanna ${ }^{1}$, Maria Angélica Souza ${ }^{1}$, Arnaldo Moura ${ }^{2}$, \\ João Meidanis ${ }^{1,2}$, Gabriel A. Costa Lima ${ }^{4}$ and Renato P. Bossolan ${ }^{3}$
}

Received July 22, 2014 / Accepted May 2, 2015

\begin{abstract}
The problem of choosing from a set of projects which ones should be executed and when they should start, depending on several restrictions involving project costs, risks, limited resources, dependencies among projects, and aiming at different, even conflicting, goals is known as the project portfolio selection (PPS) problem. We study a particular version of the PPS problem stemming from the operation of a real power generation company. It includes distinct categories of resources, intricate dependencies between projects, which are especially important for the management of power plants, and the prevention of risks. We present an algorithm based on the GRASP meta-heuristic for finding better results than manual solutions produced by specialists. The algorithm yielded solutions that decreased the risk by $47 \%$, as measured by the company's standard methodology.
\end{abstract}

Keywords: project portfolio management, power generation, optimization, scheduling.

\section{INTRODUCTION}

Decision makers are usually confronted with the problem of choosing the best portfolio of projects from a large set of available opportunities, according to a combination of criteria and a limited amount of resources over time. A project portfolio consists of a subset of the given projects, together with a schedule for their execution over a given period of time, and in such a way that all restrictions are obeyed. In this paper, we call this the project portfolio selection (PPS) problem. There are several formulations for the PPS problem [7] depending on specific constraints, objective functions, resource availability, labor policies, and timing assumptions. It may be possible to find many viable portfolios, but the main problem is to find one that is the best for a given set of conditions.

\footnotetext{
*Corresponding author.

${ }^{1}$ Scylla Informatics, Campinas, SP, Brazil. E-mails: cleber@ @sylla.com.br; cleber.mira@gmail.com

${ }^{2}$ Institute of Computing, University of Campinas, Campinas, SP, Brazil.

${ }^{3}$ AES-Tietê, Bauru, SP, Brazil.

${ }^{4}$ Mechanical Engineering Department, University of Campinas, Campinas, SP, Brazil.
} 
The single-objective function version of the PPS problem is a classical optimization problem [4, $2,3,21]$ with applications in finances [6], research and development [13, 22, 24], software development [18], and among many other fields. There is an extensive literature describing several approaches to model PPS problem variants [11,1]. Although not as studied as the single-objective function version, the multi-objective version of the PPS problem has gained increasing interest in recent years $[7,8,12,25,27]$.

In [5] the authors propose a multi-step approach to what they name the portfolio management problem. The approach focuses on three main factors relevant to the portfolio problem: value maximization, risk minimization and strategic alignment. It also has a cross-listing of other works and a number of important portfolio management concepts they deal with.

A major Swiss utility is analyzed in [17]. Mean-variance portfolio theory is used to deal with current and possible future generation mixes in order to find efficient frontiers. A costs analysis is then performed taking into account physical boundaries in order to translate the obtained portfolio allocations into required installed capacities.

In [20], risk management is treated as an important and essential part in the decision making process used by power generation companies. The authors use both physical and financial trading approaches to maximize profit potentials. Their methods take into consideration several risk factors, and are based on price risk and delivery risk. Bilateral contracts are formulated as general portfolio optimization problems with a risk-free asset and $n$ risky assets. Historical data from the electricity market is used to demonstrate the approach.

In the PhD Thesis [28], the author analyses investments into new electricity generating capacities under climate policy uncertainty, using conditional Value-at-Risk techniques. The investment problems are modeled as optimal portfolio selection problems. Differences between standard Markowitz portfolio frameworks and portfolio optimizations based on conditional Value-a-Risk are discussed, and results for real data are presented and compared.

Further discussion about other approaches to solving the single- and multi-objective versions of the PPS problem is also available [23].

Since our problem comes from a real case in the industry, with many specificities, none of the published approaches applies directly to it.

In Section 2 we outline the main characteristics of the particular PPS problem studied here. In Section 3 we present the problem input and describe the parameters needed to model and solve the problem. Section 4 describes the problem constraints. We discuss in Section 5 some optimization goals. Section 6 presents a heuristic to find good solutions for the variant of the PPS problem treated here. We show the results of experiments in Section 7, and summarize in Section 8 .

\section{Problem Description}

We describe and develop a solution for a specific version of the PPS problem according to the real case occurring at an electrical power generation company, in Brazil. Most utility companies will have a similar problem. 


\subsection{Projects}

A power generation company is periodically confronted with the selection and scheduling of projects that contribute to achieve diverse, usually conflicting, objectives such as:

1. Comply with government regulations;

2. Minimize risk exposures;

3. Increase return over investments;

4. Reduce operation costs;

In this power generation company, there are two kinds of projects:

1. Risk management projects: these projects are responsible for guarding against and mitigating risks that might arise during the power generation operation. They impact operational tasks, as well as other activities such as the refurbishing of installed equipment, the acquisition of new equipment, or the improvement of maintenance procedures. They can be scheduled with a certain amount of freedom, respecting a number of operational constraints.

2. Non-risk management projects: these are projects that do not contribute to the prevention or mitigation of risks. Examples are projects for the acquisition of office supplies, of personnel safety equipment, or of employee uniforms, among others. Non-risk management projects also include projects that help the company reach its strategic goals, e.g., the construction of a new power plant, or projects that attend exclusively to regulatory demands, such as environmental legislation concerning reservoir area, production capacity, and power availability. Due to their nature, projects of this kind cannot be scheduled at will by the decision maker; they must follow a strict, predefined schedule.

The selected projects must be scheduled to start at specific months within a period of time called the planning horizon $(\mathrm{PH})$. The extent of the planning horizon, denoted by $T$, is typically 60 months in our case study. Non-risk management projects are considered mandatory, that is, they must start at a prescribed initial month within the PH. Risk management projects are not mandatory, which means that their specific characteristics - such as costs, risk guarding potential, and interdependencies - are scrutinized by a decision maker, who decides if, and when, to schedule them for execution.

Projects are executed under the supervision of an hierarchy of administrative units. At the top of this hierarchy, we have divisions. Each division is formed by units called areas. An area can be a purely administrative unit, like an information and technology center or a general operations center. Alternatively, an area can be an operational unit, such as a power plant. Knowing which power plant will supervise a project's execution might impact its scheduling, since restrictions may limit the number of projects that can be executing simultaneously at a given power plant. 


\subsection{Warning points}

The generation of electricity is a complex process. As such, several undesired events might occur e.g., equipment failure, violation of government regulations, or accidents. Then, prevention against the occurrence of undesired events is a top priority and there is a consolidated process to achieve this control, consisting of the following steps:

1. Elicit warning points, that is, identify specific conditions that may cause undesired events. Warning points are then used to guide specialists when they elaborate measures to prevent the occurrence of undesired events.

2. Assign to each warning point an amount of risk according to a standard set of criteria. Such criteria might include the probability of occurrence of their associated undesired events, how severe a safety hazard it can be, the severeness of occasional environment damages that might follow, or threats to the company image, among others. The amount of risk should be proportional to the impact the occurrence of the corresponding undesired events may cause, if the warning point is not controlled. Warning points are classified into two risk categories, namely, critical and non-critical. The non-critical category includes warning points with either a tolerable or moderate risk. The critical category includes warning points with an intolerable risk. The risk category is assigned by expert personnel and will be seen, in the sequel, to impact the scheduling of a project.

3. Devise projects that control the warning points, in order to prevent or mitigate the impact of the associated undesired events. Usually, a warning point is controlled by a group of projects. In our present modeling, all projects in a group are taken to contribute equally for controlling the risk associated to the warning point. According to the available data, and following field engineers advice, this was deemed reasonable in a first approach. Notice that a project may belong to more than one group of projects, and therefore it may contribute to control the risk of several warning points. The total risk controlled by a project is obtained by summing all the shares of risk the project controls in all groups it belongs to.

In this PPS problem variant we focus on controlling the risk assigned to warning points. The risk associated to a warning point can be considered completely averted only after all projects in its controlling group have been completely executed. Moreover, high-risk warning points must have their associated risk completely controlled by the end of a given deadline, which is a specific month along the PH. Only high-risk warning points have an assigned deadline.

\subsection{Power plants and generating units}

The power generation process is organized around geographical locations. Each location has to produce a minimum amount of electrical power to comply with government regulations. The power production in a certain location is the sum of the electrical power generated by several power plants. 
Table 1 - The list of symbols used throughout the text.

\begin{tabular}{ll}
\hline$T$ & size of planning horizon (PH) in months \\
$I$ & set of projects \\
{$[n]$} & interval $1,2, \ldots, n$ \\
$d_{p}$ & duration (in months) of project $p$ \\
$I_{P}$ & set of projects in portfolio $P$ \\
$m_{p}$ & starting month of project $p$ in portfolio $P$ \\
$e_{p}$ & last month of execution of project $p$ \\
$l_{p}$ & lead time of project $p$ \\
$p_{m}$ & prescribed initial month for project $p$ in the manual solution \\
$G_{w}$ & group of projects controlling warning point $w$ \\
$W$ & set of all warning points \\
$f_{w}$ & last month of the tardiest project controlling warning point $w$ \\
$D_{w}$ & deadline associated to high-risk warning point $w$ \\
$Q$ & the set $\{C A P E X, O P E X\}$ \\
$r_{p, m}$ & resource consumption of project $p$ in the $m$-th month of its duration \\
$P_{t, q}$ & set of projects of class $q$ active in month $t$ \\
$c_{P, t, q}$ & resource consumption of class $q$ on month $t$ for portfolio $P$ \\
$C_{P, y, q}$ & yearly version of $c_{P, t, q}$ above \\
$S_{y, q}$ & resources available for class $q$ and year $y$ \\
$g(u, t)$ & number of generating units halted at plant $u$ on month $t$ \\
$g_{L}(u, t)$ & same as $g(u, t)$ but due to long-term projects \\
$R_{w}$ & risk of warning point $w$ \\
$W_{P}$ & set of warning point effectively controlled by portfolio $P$ \\
$R(P)$ & area under the risk curve for portfolio $P$ \\
$V_{I}$ & set of viable portfolios for instance $I$ \\
\hline &
\end{tabular}

Each power plant has a specific location associated to it. Besides their location, power plants are also associated to divisions, for management purposes. Both associations, into locations and divisions, have an influence on the operation of power plants. This influence will be reflected on the problem restrictions.

A power plant hosts a certain number of generating units. Periodically, generating units may be halted for several reasons, including seasonal events and maintenance operations. The execution of maintenance projects may be affected by restrictions on the simultaneous halting of generating units. For instance, once a generating unit goes down for maintenance, other units in the same power plant might have to be kept working, which might forbid the simultaneous execution of other maintenance projects on the remaining generators at that power plant. The impossibility of simultaneously executing certain maintenance projects establishes an exclusion dependency between maintenance projects. Only maintenance projects that cause the halting of generation units are affected by excluding dependencies. 
The PPS problem is a difficult problem [9] - NP-hard in the computer science jargon. Given its difficulty, we decided to approach this particular PPS problem using an heuristic of easy implementation and that provides good results.

\section{PROBLEM INPUT}

The input data provided by the company is composed by a set of projects, a set of warning points, a planning horizon, $\mathrm{PH}$, the availability of resources along the $\mathrm{PH}$, and other data about power plants and generating units.

The planning horizon is always taken to be five years, that is, its extent $T$ is set to 60 months. However, the portfolio expected as a solution for the problem has an execution horizon (EH) of ten years $(2 T)$ : the first five years are the planning horizon, while the final five years serve to accommodate projects that, although initiated before the final month on the $\mathrm{PH}$, will finish after the end of the $\mathrm{PH}$. With a duplication of the $\mathrm{PH}$ period we can register how much a project that terminates beyond the last month in the $\mathrm{PH}$ will contribute to reduce the portfolio's overall risk. Duplicating the planning horizon is enough, since no project execution is longer than five years.

\subsection{Available resources}

For administrative and fiscal reasons, and government regulations, resources must be classified into two categories: operational expenditures (OPEX) and capital expenditures (CAPEX). The costs of each project are always entirely of only one of these two categories.

Resource availabilities are presented in two series of five annual amounts. The first series designates annual resource availabilities for CAPEX expenditures, and the second one represents annual values for OPEX expenditures. Annual resource availabilities for years after the PH are replicated from the given resource availability for the first five years, in both categories.

\subsection{Projects}

A project is defined by the following set of parameters and constraints:

1. Maintenance duration: for maintenance projects, this parameter indicates whether the project's duration is short-term (S) or long-term (L). For non-maintenance projects, the indication is $\mathrm{N}$.

2. An area: an identification of the administrative unit or power plant associated to a project. The administrative units include an "operation and management center" (COG), and an "information technology center" (IT). The power plants include: AGV, BAB, BAR, CAC, EUC, IBI, LMO, MOG, NAV, PRO, SJS, SJQ. See Table 2.

3. Generating unit identifier: for maintenance projects, the identifier of the generating unit in the corresponding power plant that will be affected by the project's execution. A maintenance project affects no more than one generating unit. 
4. Halting start month: for a maintenance project, the halting start month indicates from which month, counting from its first execution month, the project will halt the corresponding generating unit.

5. Halting duration: for a maintenance project, the halting duration informs for how long, in months, the generating unit affected by the project will be halted.

6. A manually selected initial month at which the project is scheduled to start. The set of all projects, together with the corresponding initial months, forms a portfolio, dubbed the initial portfolio. This portfolio was manually constructed by expert engineers at the company, and can be used as a yardstick for comparison against other solutions.

7. A project lead time is the number of months, counting from the beginning of the $\mathrm{PH}$, after which the project can be scheduled to start.

8. A project kind, which can be risk management or non-risk management. The project kind is used to indicate whether a project is mandatory, and therefore must start at its assigned initial month. Non-risk management projects are mandatory.

9. A resource classification designating the kind of resource it consumes: OPEX or CAPEX. A project can consume resources from one category only.

10. A sequence of values, also referred to as the project's costs, describing the amount of resources the project will consume in each month during its execution, starting with the initial month. The duration of a project is the number of months necessary for its execution.

\subsection{Warning points}

Warning points are specified by the following parameters:

1. An identifier: a unique, numeric identifier.

2. A risk measure: a rational number indicating the risk associated to the warning point.

3. Group of projects: a list of project identifiers that defines the group of projects that controls this warning point. Note that some projects may not belong to any group and, therefore, they do not contribute to risk control, e.g., a project for office supplies acquisition.

4. A risk category: a qualitative classification of the risk into two categories: critical or noncritical.

5. A deadline: the latest month at which the warning point must be completely controlled. The deadline is present for high-risk warning points only.

\subsection{Power plants and generating units}

The power generation system comprises 12 power plants. Each plant hosts a certain number of generating units and must adhere to specific regulations for their operation, according to their 
geographical locations and administrative divisions. Table 2 summarizes the administrative hierarchy of the company generation system, including the information about divisions, areas and power plants, geographical locations and the number of generating units in power plants.

The following parameters are provided for each power plant:

1. Plant identifier: a unique, three-letter code identifier;

2. Generating units: the number of generating units in the power plant;

3. Division: the division responsible for managing the plant;

4. Geographical location: the geographical location the plant resides in.

Table 2 - Administrative hierarchy of the company system. The first column gives the administrative division the plant is subordinate to. The second column gives the area. The third column specifies the power plant location. The fourth column shows the number of generating units at the corresponding power plant.

\begin{tabular}{|cccc|}
\hline Division & Area/Plant & Location & $\begin{array}{c}\text { Generating } \\
\text { Units }\end{array}$ \\
\hline COG & COG & - & - \\
\hline IT & IT & - & - \\
\hline AGV & AGV & AV & 6 \\
\hline \multirow{2}{*}{ PRO } & NAV & NAPI & 3 \\
& PRO & NAPI & 3 \\
\hline \multirow{3}{*}{ BAR } & BAB & BBB & 4 \\
& BAR & BBB & 3 \\
& IBI & NAPI & 3 \\
\hline \multirow{4}{*}{ LMO } & CAC & RP & 2 \\
& EUC & RP & 4 \\
& LMO & RP & 2 \\
& MOG & RMG & 2 \\
& SJS & RJM & 2 \\
& SJQ & RJM & 1 \\
\hline
\end{tabular}

\section{CONSTRAINTS}

In this section we list all constraints for this particular version of the PPS problem. Notice that this PPS version is not a linear problem as can be seen by the nature of its constraints like, for instance, the halting restriction expressed by Eq. (13). This does not pose a difficulty to our modeling, since we developed a variant of a GRASP meta-heuristic to solve the problem and, as is well known, a GRASP meta-heuristic can cope with nonlinearities.

Let $I$ be the set of projects in an input instance for the PPS problem, let $W$ be the set of warning points in that instance, and let $T$ be the associated planning horizon, measured in months. We 
will always assume that $T$ comprises an integral number of years, that is, it is a multiple of 12 . For each project $p \in I$, we will indicate its duration, in months, by $d_{p}$. For ease of reference, we may write $[n]$ for the interval $[1, n]$, where $n$ is a positive integer. For a complete list of symbols used throughout the text, see Table 1.

A portfolio $P$ is a set of pairs $(p, m)$ where $p$ is a project in $I$ and $m$ is the month in which project $p$ is scheduled to start its execution, that is, $P \subseteq I \times[T]$. We will indicate by $I_{P} \subseteq I$ the set of projects present in portfolio $P$.

A portfolio $P$ is valid if it satisfies the following set of constraints.

Consistent scheduling. A project cannot be scheduled to start at two different months.

$$
\text { If }\left(p, m_{1}\right) \in P \text { and }\left(p, m_{2}\right) \in P \text { then } m_{1}=m_{2}, \quad \text { for all } p \in I_{P} .
$$

This means that a valid portfolio $P$ is in fact a function $P: I_{P} \mapsto[T]$. From now on, we will let $m_{p}=P(p)$ indicate the starting month and $e_{p}=m_{p}+d_{p}-1$ will be the last execution month of project $p$, when $p \in I_{P}$.

Lead Time. A project $p$ cannot be scheduled to start before its lead time $l_{p}$ has passed. Then,

$$
m_{p}>l_{p}, \quad \text { for all } p \in I_{P} .
$$

Mandatory projects. A mandatory project $p$ should always start at its prescribed initial month, $p_{m}$. Hence,

$$
\left(p, p_{m}\right) \in P, \quad \text { for all mandatory projects } p \in I \text {. }
$$

Intolerable warning points. A warning point whose risk category is ranked as high-risk is an intolerable warning point and have a deadline associated to it. Each intolerable warning point must be completely controlled by the end of its deadline. This means that all projects in the control group of any intolerable warning point must be completed at that deadline Let $G_{w} \subseteq I$ the group of projects controlling warning point $w$. Recall that $W$ is the set of all warning points in $I$. Then we must have

$$
G_{w} \subseteq I_{P}, \quad \text { for all intolerable warning points } w \in W .
$$

This restriction being satisfied, define

$$
f_{w}=\max _{p \in G_{w}}\left(m_{p}+d_{p}-1\right) .
$$

We require, further, that

$$
f_{w} \leq D_{w}, \quad \text { for all intolerable warning points } w \in W,
$$

where $D_{w}$ stands for the deadline associated to an intolerable warning point $w$.

We point out that the concept of warning points as described in this paper is specific to the risk management practices found in the company's case under study. A more standard view on risk management terminology can be found in references $[16,15]$. 
Limited resources. Each project demands a certain amount of monthly resources from the month it starts until the last month of its execution. All resources consumed by a project are of the same kind as the resource classification associated to the project. The total yearly resource consumption of a certain kind, counting all projects that are under execution in that year, should not surpass the given amount of available resources of that kind and for that year.

The set of resource classifications is $Q=\{\mathrm{CAPEX}, \mathrm{OPEX}\}$. For any project $p \in I$, let $r_{p, m}$ be the resource consumption of project $p$ during the $m$-th month of its execution $\left(1 \leq m \leq d_{p}\right)$. For a portfolio $P$, we collect in $P_{t, q}$ the set of all projects of class $q \in Q$ that are active at month $t \in[2 T]$, that is $P_{t, q}=\left\{p \in I_{P} \mid t \in\left[m_{p}, e_{p}\right]\right\}$. Then

$$
c_{P, t, q}=\sum_{p \in P_{t, q}} r_{p, t-m_{p}+1}, \quad t \in[2 T], q \in Q, P \text { a portfolio, }
$$

gives the resource consumption at month $t$ for all active projects of class $q$ that are already scheduled at portfolio $P$. We can now collect the consumption of resources of a class $q$ in a year $y$ for a portfolio $P$ :

$$
\begin{gathered}
C_{P, y, q}=\sum_{t \in M_{y}} c_{P, t, q}, \quad y \in[1, T / 12], q \in Q, \\
M_{y}=[12 y-11,12 y], P \text { a portfolio. }
\end{gathered}
$$

So, if $S_{y, q}$ is the total amount of resources of class $q$ available for year $y$, this restriction imposes the constraint

$$
C_{P, y, q} \leq S_{y, q} \quad \text { for all } y \in[1, T / 12], q \in Q, P \text { a portfolio. }
$$

We describe next the exclusion dependency constraints involving maintenance projects. We note that the only reason for halting a generating unit is the execution of a maintenance project that affects it. A maintenance project causes the halting of a generating unit for a limited period of time during its execution. That period is defined by the project's halting start month and halting duration.

Halting restrictions by geographical location. Restrictions involving the halting of generating units in some locations stem from engineering considerations. Let $g(u, m)$ denote the number of generating units halted at plant $u$ and month $m$ in a given portfolio, while $g_{L}(u, m)$ denotes the number of generating units halted at plant $u$ and month $m$ due to long-term maintenance projects. Referring to Table 2, the restrictions are as follows:

1. In the RP location, in any given month, if a plant has two or more generating units halted, the other plants cannot have any generating units halted. More formally, we have

$$
\left(g\left(u_{1}, m\right) \geq 2\right) \rightarrow\left(g\left(u_{2}, m\right)=0\right),
$$

for $m \in[2 T]$ and $u_{1}, u_{2} \in\{C A C, E U C, L M O\}$, with $u_{1} \neq u_{2}$. 
2. In the BBB location it must be observed that:

- In any given month, if a plant has two or more generating units halted, the other plants cannot have any generating units halted. Thus,

$$
\left(g\left(u_{1}, m\right) \geq 2\right) \rightarrow\left(g\left(u_{2}, m\right)=0\right)
$$

for $m \in[2 T]$ and $u_{1}, u_{2} \in\{B A B, B A R\}$, with $u_{1} \neq u_{2}$.

- In any given month, at most two generating units from the BAR plant can be down. Thus,

$$
g(B A R, m) \leq 2, \quad \text { for } m \in[2 T]
$$

- In any given month, two generating units from the BAB plant can be down simultaneously, but only if none of the BAR plant generating units are halted. Thus,

$$
(g(B A B, m)=2) \rightarrow(g(B A R, m)=0), \quad \text { for } m \in[2 T] .
$$

3. In the NAPI location, at most two generating units can be down at any given month. Thus,

$$
g(N A V, m)+g(P R O, m)+g(I B I, m) \leq 2, \quad \text { for } m \in[2 T] .
$$

4. In any given month, in the AV location, we can have at most two halted generating units due to long-term maintenance projects. Thus,

$$
g_{L}(A G V, m) \leq 2, \quad \text { for } m \in[2 T] .
$$

Halting restrictions at the AGV plant. In any given month, if two or more generating units from the AGV plant are down, then no generating unit from the BAB, NAV, or PRO plants can be halted, that is

$$
\begin{aligned}
& (g(A G V, m) \geq 2) \rightarrow(g(B A B, m) \\
= & g(N A V, m)=g(P R O, m)=0), \quad m \in[2 T] .
\end{aligned}
$$

Halting restrictions by division. In any given month, no more than three generating units in the same division can be halted. Let $D$ be the set of divisions in the company electric power generation system, as given by Table 2 . For each division $d \in D$, let $U_{d}$ be the set of power plants in $d$. We must have

$$
\sum_{u \in U_{d}} g(u, m) \leq 3, \quad \text { for } m \in[2 T], d \in D .
$$

For instance, if no other constraint takes effect, then we are allowed to halt one generating unit from each of the BAB, BAR, and IBI power plants, in the BAR division. 
In a practical scenario, real problems of this complexity might involve uncertainties, like a scheduled project not terminating its execution or having its termination delayed due to unanticipated events $[19,14]$. This is not the case with the present PPS problem, however. But it should be noted that the concept of risk, as managed by AES in this PPS problem, is not usual, as discussed in Subsection 2.1, and it is also not related to these kinds of uncertainties. Therefore, approaches that treat the more common kinds of uncertainty, as mentioned, are not directly applicable to this version of the PPS problem.

\section{OBJECTIVE FUNCTION}

A set of optimization goals can be proposed for the PPS problem depending on which aspect - costs, human resources, risks - one wants to focus upon. While planning and constructing a portfolio, the company decision makers focus their efforts on reducing the risk resulting from all uncontrolled warning points. For that, they try to judiciously select and schedule projects of the risk management kind. The objective function of the PPS problem studied here should also reflect the best choice of scheduling projects in order to guard against uncontrolled warning points. So, in this version of the PPS problem, we focused on the optimization goal of reducing the risk of uncontrolled warning points in a portfolio.

As we advance through the EH time frame, projects terminate and warning points reach a controlled state. When that happens, the total stock of unguarded risks decreases until it reaches a minimum, ideally 0 , at month $2 T$ or earlier. The area under the risk curve summarizes in a single number two of the most relevant aspects of a portfolio: the amount of controlled risk and when the risk is averted. That is, the earlier a warning point is effectively controlled, and the larger is the risk value associated to the warning point, the better is the corresponding portfolio. Therefore, we set the objective function to minimize the area under the risk curve associated to the portfolio.

Recall that $W$ is the set of all warning points in an input instance $I$ to the PPS problem. Let $R_{w}$ denote the risk associated to warning point $w \in W$. For a portfolio $P$ we let $W_{P} \subseteq W$ be the set of warning points that are effectively controlled in $P$. Then, the area under the risk curve for $P$ is

$$
R(P)=2 T \sum_{w \in W} R_{w}-\sum_{w \in W_{P}} R_{w}\left(2 T-f_{w}\right),
$$

where $f_{w}$, given by Eq. (5), is the first month when warning point $w$ is finally controlled (see Figure 1).

For a given input instance $I$ to the PPS problem, let $V_{I}$ be the set of all viable portfolios that can be obtained from $I$. The goal, then, is to construct a portfolio $P^{\star}$ such that $R\left(P^{\star}\right)$ is minimum among $\left\{R(P) \mid P \in V_{I}\right\}$. In the next section we describe a heuristic strategy that searches for a portfolio $P$ that, although not guaranteed to attain $R(P)=R\left(P^{\star}\right)$, improves considerably its risk measure when compared to other portfolios manually constructed by expert engineers. 


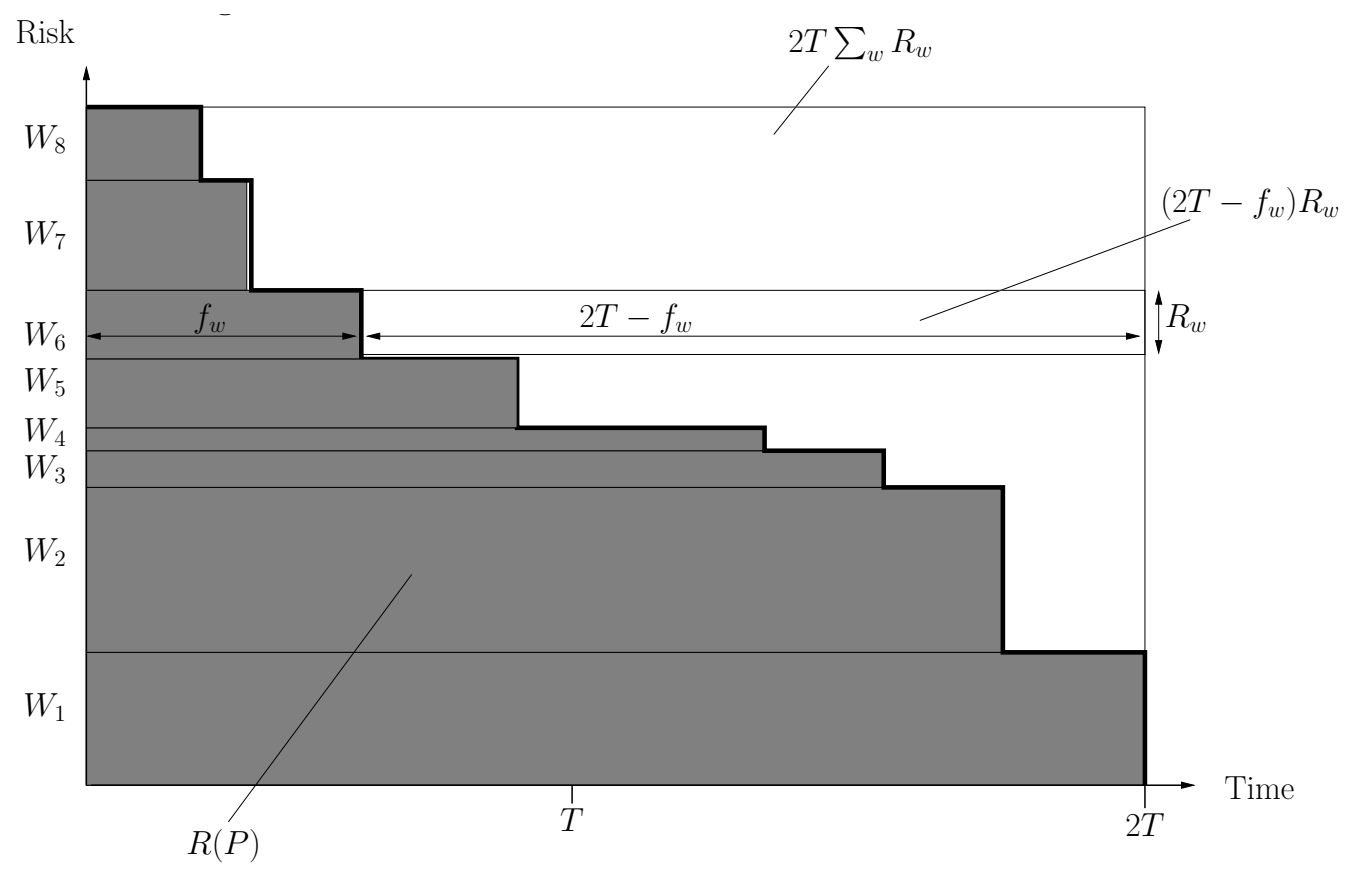

Figure 1 - Illustration of the area under the risk curve.

\section{A GRASP VARIANT FOR THE PPS PROBLEM}

We describe a variant of the GRASP meta-heuristic for finding solutions for the PPS problem treated here. Those solutions will prove to be better than the manually produced solutions provided by specialists. GRASP is a multi-start meta-heuristic that generates good quality solutions for many combinatorial optimization problems [26, 10], besides being of fast implementation, being easily comprehensible, and not requiring restrictive assumptions of mathematical optimization models; e.g. linear constraints. Thus, the GRASP meta-heuristic is suitable as a first approach to try and find better solutions than the manual ones. The differences between our GRASP variant and the original meta-heuristic are explained as follows.

The algorithm repeats two phases:

(i) A construction phase in which a feasible solution is obtained; and

(ii) a local search phase in which the algorithm finds a local optimal solution by iteratively checking whether there is a better solution in a neighborhood of the current solution.

Several initial feasible solutions are constructed and improved upon until the best overall solution is then chosen. We implemented a variant of this basic strategy. First, a pool of viable portfolios is constructed. Then, we apply the local search procedure to the $\beta$ best solutions in the pool, where $\beta$ is a fraction of the pool size. This cycle is repeated a number of times, and the best solution is finally returned. 
The pseudo-code for the GRASP routine is presented as Algorithm 1. In the next two subsections the construction and local search phases are discussed more fully. The choices of parameters appearing in the algorithms are discussed in more detail in Section 7.

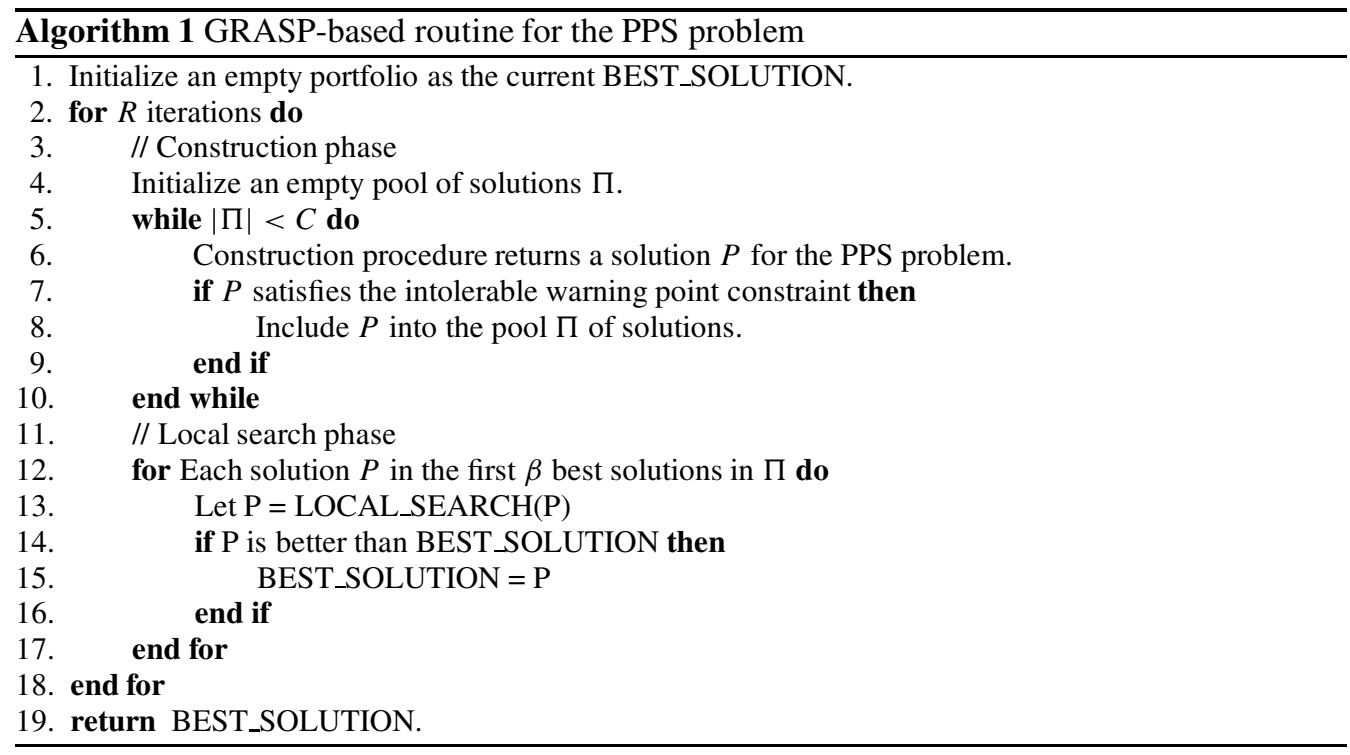

\subsection{The Construction Phase}

The first phase in our GRASP-based algorithm consists in the repeated execution of a construction procedure. As shown in Algorithm 1, the construction procedure produces a solution and checks whether it satisfies the intolerable warning point constraint as expressed by Eqs. (4) and (6). These constraints require that all projects that control warning points must be scheduled in such a way that the corresponding warning points are totally under control before their deadlines are reached. If the portfolio is valid, then it is included in the pool of constructed solutions; otherwise, it is discarded. Feasible solutions are inserted into the pool until its capacity $C$ is reached. Notice that the same solution might be included into the pool more than once. This does not seem to be a problem, however, and, moreover, since the solutions are relatively large structures, checking for equality is costly and does not seem to be worth the effort. The best $\beta$ of the constructed solutions in the pool are chosen as input solutions for the local search phase.

Two kinds of construction procedures were considered: an adaptive one and a non-adaptive one. We start with the non-adaptive procedure.

\subsubsection{The non-adaptive procedure}

The non-adaptive heuristic was devised as a simple procedure using two candidate lists of pairs $(p, m)$ of projects $p \in I_{P}$ and potential starting months $m \in\left[l_{p}+1, \min \left(M D_{w}-d_{p}+1, T\right)\right]$, where $M D_{w}$ is the minimum among all deadlines associated to all intolerable warning points $p$ helps to control. If a project $p \in I_{P}$ does not control any warning points, we consider all pairs $(p, m)$ with $m \in[T]$. The lists are then sorted according to a suitable key parameter. The key 
parameter associated to a pair $(p, m)$ should measure the gains obtained by scheduling project $p$ at month $m$, based not only on $p$ 's ability to control risk, but also on its total cost. Notice that a project with a large value of controlled risk may be less cost-effective in terms of controlled risk per unit of cost than another project [23]. Notice also that we use two candidate lists instead of a single list, as is standard in the construction phase of a GRASP routine. This allowed us to classify the projects into two priority categories.

Let $I$ be the set of projects in an input instance for the PPS problem, with planning horizon $T$, and warning point set $W$. In order to assess the cost-effectiveness of a pair $(p, m) \in I \times[T]$, we compute $b_{p, m}$, the benefit of the pair $(p, m)$. Let $R_{p}$ be the sum of warning point risk shares project $p$ contributes to control, computed over all warning points in $W$, and recall that $r_{p, t}$ gives the resource consumption of project $p$ during its $t$-th month of execution. We let

$$
b_{p, m}=R_{p}\left(\left(2 T-m-d_{p}+1\right) / \sum_{t=1}^{d_{p}} r_{p, t}\right),
$$

so that $b_{p, m}$ expresses the amount of risk project $p$ helps to control, weighted by how long that contribution is effective per unit of cost.

The portfolio construction procedure initially pre-processes the input data to schedule all mandatory projects in $I$, which are then removed from $I$. Next, all pairs $(p, m) \in I \times[T]$, have their benefit calculated, and the two candidate pair lists are built and sorted in non-increasing order of benefit. The high-risk sorted pairs (HRSP) list involves pairs $(p, m)$ where project $p$ contributes to control high-risk warning points. The sorted pairs (SP) list involves all the remaining pairs in $I \times[T]$. We split the standard GRASP candidate list in two parts in order to give pairs in the HRSP list a higher priority over the SP list, since the corresponding projects help control high-risk warning points.

The algorithm proceeds to construct a portfolio by iteratively choosing a pair $(p, m)$ from one of the two candidate lists. The next pair is chosen from the HRSP or the SP lists with probabilities $\eta$ and $1-\eta$, respectively. Once the list to choose the next pair from is determined, the next pair $(p, m)$ is randomly chosen from among the first $k$ pairs in that list. Then pair $(p, m)$ is included in the portfolio being constructed if, by including it, the new portfolio does not violate any of the following local constraints:

- "consistent scheduling", Eq. (1);

- "lead time", Eq. (2);

- "limited resources per year", Eq. (9); and

- "halting restrictions", Eqs. (10-17).

Observe that, at each iteration, as a new pair $(p, m)$ is included, the available resources for each year decrease, reflecting the resource usage of project $p$, and the cycle repeats until no pair $(p, m)$ can be included in the portfolio.

At the end of the construction procedure the portfolio generated is returned. 
We summarize the non-adaptive procedure used in the construction phase as Algorithm 2.

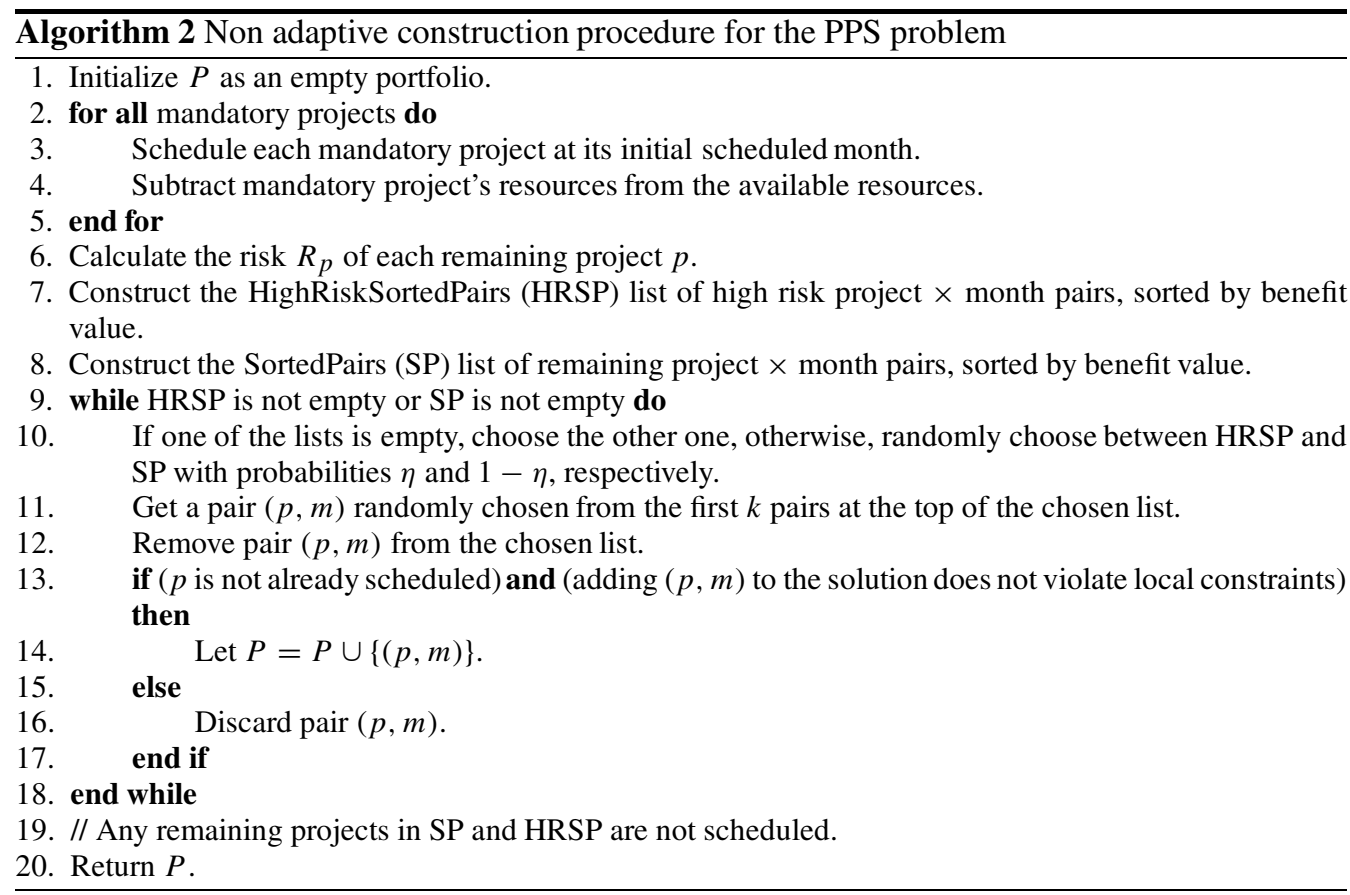

\subsubsection{The adaptive procedure}

An adaptive version of the construction procedure involves the recalculation of the benefits of each remaining pairs in the candidate lists, after a new pair is included in the portfolio being constructed. After recalculation, the lists are sorted again.

The benefit function given by Eq. (19) is a first attempt to express the cost-effectiveness of scheduling a project at a certain month. However Eq. (19) does not take into account the impact on available resources caused by scheduling a new project. Given a portfolio $P$, we want to measure the impact of including a new pair $(p, m)$ in $P$ by estimating which fraction of the presently available resources a new pair $(p, m)$ will consume, at each month project $p$ is in execution. Furthermore, since costs are grouped annually, we need to sum $p$ 's resource consumption over all months it is active in each year.

We denote by $M(p, m, y)$ the set of months project $p$ will be active during year $y$, if it starts at month $m$. Note that, in this case, $p$ would end its execution at month $e_{p}=m+d_{p}-1$. Note also that $12(y-1)+1$ is the first month of year $y$, and $12 y$ is its last month. We then have, for $y \in[1,2 T / 12]:$

$$
M(p, m, y)= \begin{cases}\emptyset, & \text { if } 12 y<m \text { or } e_{p}<12(y-1) \\ {\left[m, \min \left\{e_{p}, 12 y\right\}\right],} & \text { if } 12(y-1)<m \leq 12 y \\ {\left[12(y-1)+1, \min \left\{e_{p}, 12 y\right\}\right],} & \text { if } m \leq 12(y-1)<\min \left\{e_{p}, 12 y\right\}\end{cases}
$$




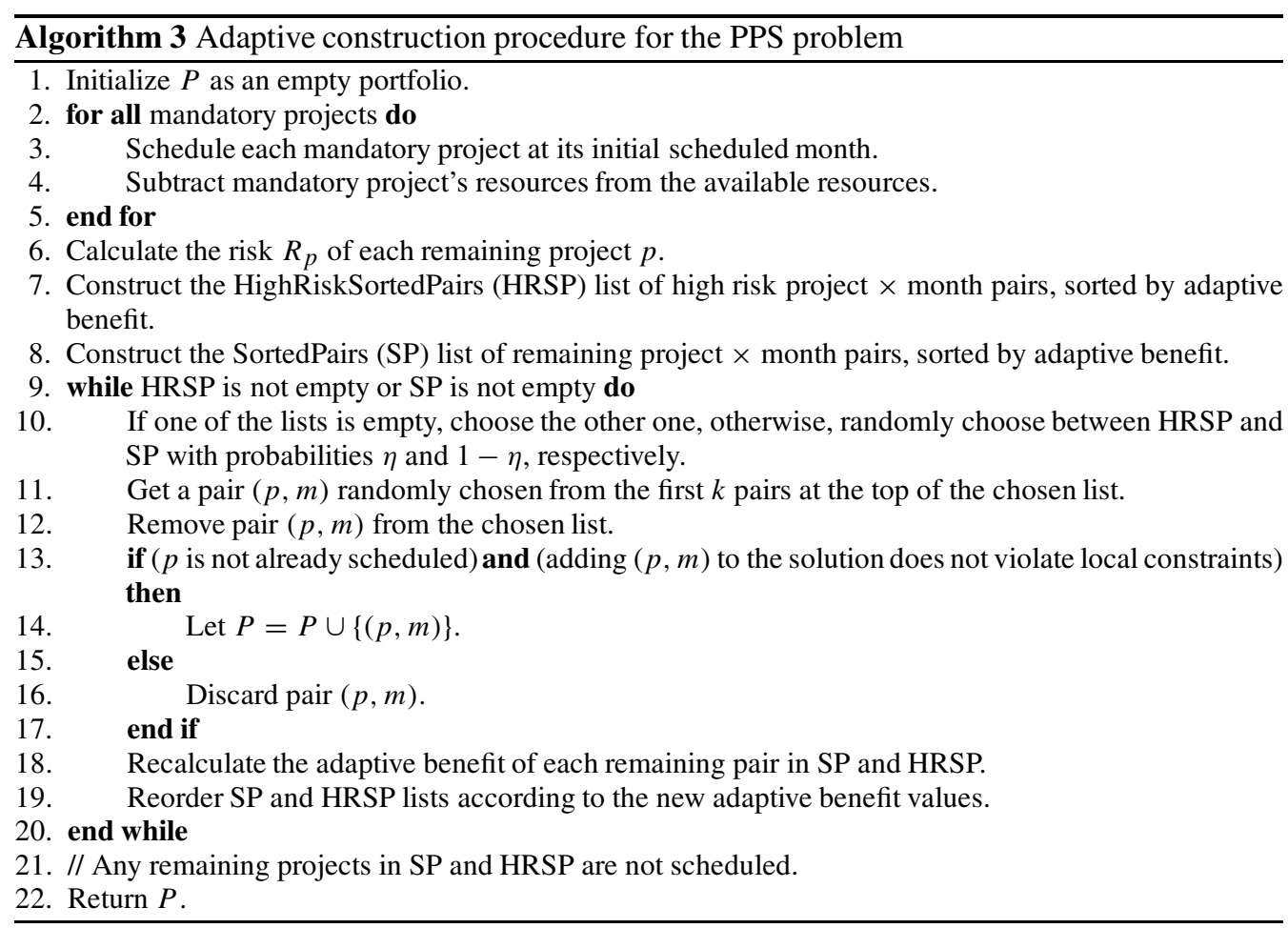

Then, if $h(p, m, y)$ gives the resource consumption of project $p$ during year $y$ when started at month $m$, we have

$$
h(p, m, y)= \begin{cases}0, & \text { if } M(p, m, y)=\emptyset \\ \sum_{j \in M(p, m, y)} r_{p, j-m+1}, & \text { otherwise. }\end{cases}
$$

Recall that, by Eq. (8), $C_{P, y, q}$ gives the total resource consumption for projects of category $q$ in a portfolio $P$ during year $y$, and $S_{y, q}$ is the total amount of resources of category $q$ in a year $y$, as given by the input instance. Then,

$$
\kappa(p, m, y)=\frac{h(p, m, y)}{S_{y, q}-C_{P, y, q}},
$$

gives the fraction of the currently available resources, at year $y$, that will be consumed by the inclusion of a pair $(p, m)$ in the current portfolio $P$, where $q$ is $p$ 's category. To avoid division by zero, when $S_{y, q}=C_{P, y, q}$, we set $\kappa(p, m, y)=0$.

The maximum fraction of yearly resource usage that a project $p$ will incur when the pair $(p, m)$ is considered for inclusion in a portfolio $P$ is, then,

$$
\rho(p, m)=\max _{1 \leq y \leq T / 12} \kappa(p, m, y) .
$$

This same idea can be used with other functions instead of the 'maximum' in Eq. (23), e.g., 'minimum', 'average', or 'sum'. 
In order to recalculate the cost-effectiveness of a project $p$ being scheduled at month $m$ in a portfolio $P$, and now taking into consideration the changes in available resources, we adopted as a measure of its effectiveness its adaptive benefit $b_{a}(P, p, m)$, defined as

$$
b_{a}(P, p, m)=R_{p}\left(\left(2 T-m-d_{p}+1\right) / \rho(p, m)\right) .
$$

We note that every project consumes a non-null amount of resources in its corresponding category. The adaptive construction procedure is shown in Algorithm 3.

\subsubsection{Comparing the construction strategies}

We performed some experiments in order to compare the adaptive and the non-adaptive construction procedures. We used as input instance the real-world based data described in Section 7.

In order to check how much the resource usage has an impact on the quality of solutions, we performed experiments using variations on the adaptive benefit $\rho(p, m)$ described by Eq. (23), namely, we used the minimum, the average and the sum of the fractions of resource usages. We also used a pool with a capacity $C$ of 100 solutions and also recorded the number of unfeasible solutions obtained until we reached full capacity. Table 3 shows the result of these experiments, where the real instance was used as input to the algorithm. The first column defines the construction strategy, the second column specifies how the fraction of yearly resource consumptions are combined, the next two columns give the best and the average values of the optimized objective function (OOF) values, respectively, the last two columns report the number of unfeasible solutions that were constructed and the running times, respectively.

Table 3 - Construction strategy, best solution, average solution value (both in $10^{6}$ risk-month units), number of solutions, running time values (in seconds) for a real-world input instance.

\begin{tabular}{|l|cccc|c|}
\hline Strategy & resource usage & best OOF & avg OOF & unfeasible solutions & Time (s) \\
\hline Non adaptive & & 0.570 & 0.638 & 0 & 74 \\
\hline \multirow{4}{*}{ Adaptive } & avg & 0.645 & 0.698 & 118 & 2343 \\
& max & 0.741 & 0.846 & 43 & 1417 \\
& min & 0.739 & 0.820 & 24 & 932 \\
& sum & 0.649 & 0.700 & 128 & 1691 \\
\hline
\end{tabular}

From Table 3, we find that the best solution found by the adaptive procedure - using the maximum resource usage in the benefit function - is around $23.4 \%$ worse than the one found by the non-adaptive procedure. In addition, the adaptive procedure finds more invalid solutions and takes considerably more time to be executed than the non-adaptive strategy. Given these limitations on the side of the adaptive procedure, we decided to adopt the non-adaptive procedure in our GRASP-based heuristic.

\subsection{Local search}

The second phase of our GRASP heuristic consists of executing a local search procedure. For a fraction of the solutions obtained in the construction phase, a distinct local search procedure is 
executed. The local search starts at the input solution and examines its neighborhood, checking whether there is a better solution in terms of the objective function, using a best improvement strategy. It iteratively repeats the search in the neighborhood of each new solution until no better solution is found. The best solution thus found is then returned. The local search procedure is shown as Algorithm 4.

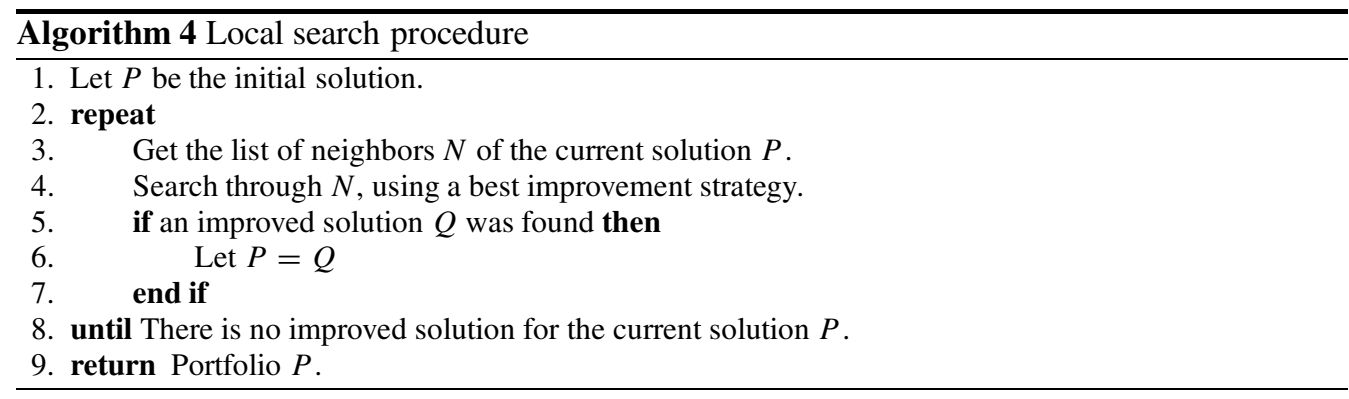

A fundamental aspect of a local search procedure concerns how the neighborhood of a solution is defined. We defined the neighborhood of a portfolio as the set of portfolios where each neighbor is obtained by re-scheduling one of its projects. If a project was originally scheduled at month $m$, a neighbor is obtained by re-scheduling it at months from $m-\Delta$ to $m+\Delta$, avoiding the current $m$, and where $\Delta$ is a fixed parameter. If re-scheduling leads to infeasibility, or to a project starting at a month beyond $T$, that neighbor is discarded. We note also that only projects whose start times are in the interval $[1, T]$ are considered for re-scheduling. Another relevant aspect of the local search is how the neighborhood is searched. We implemented a best improvement strategy, which searches through all projects in the neighborhood and selects the best one. Algorithm 5 shows the procedure to construct the neighborhood of a portfolio.

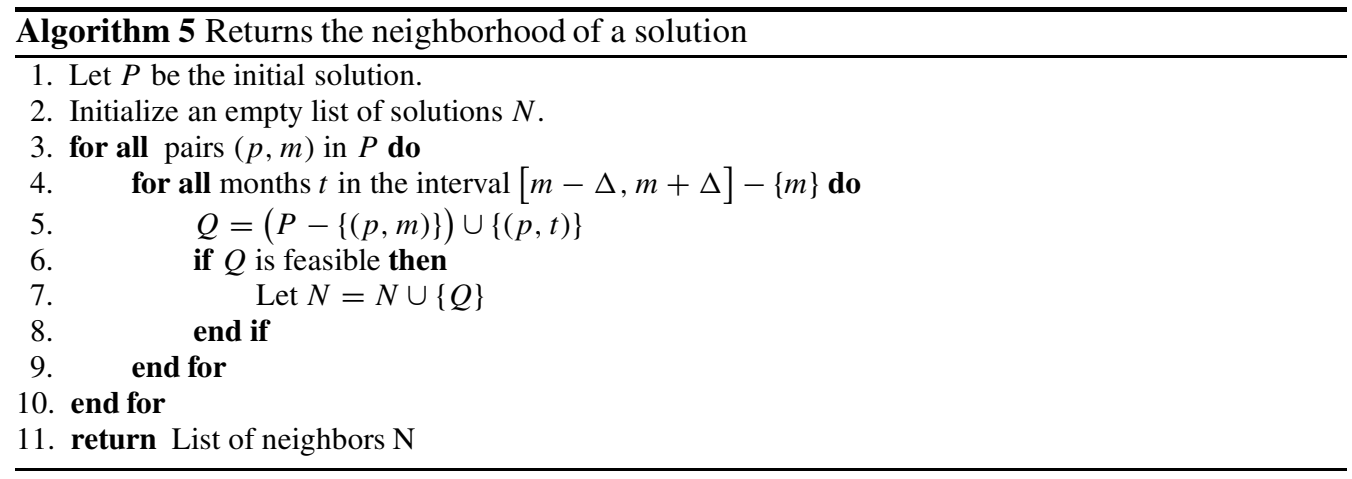

\section{EXPERIMENTS}

We performed experiments to evaluate the performance and robustness of the complete algorithm. As solution quality indicators, we chose the heuristic running-time and the objective function value. 
In the company input instance, we had 1411 projects for a planning horizon comprising the years 2013-2017. The total number of warning points were 434 and the sum of all attributed risk values was around $25 \times 10^{3}$ risk-units. Risk values were measured in a relative, dimensionless, number of "risk points". The annual costs were 1.5, 1.0, 1.7, 1.3, and 0.7, expressed in $10^{9}$ monetary units, the Brazilian real. These figures all stemmed from real values, but they were multiplied by an undisclosed constant, in order to comply with the company confidentiality policies which do not permit the real values to be disclosed in a text that is publicly accessible.

The amount of available yearly CAPEX resources was obtained by summing up the CAPEX costs of all projects in the initial manual solution that were active at each year. Yearly OPEX available resources were obtained in a similar fashion.

\subsection{Input instances}

In order to assess the indicator values and test the robustness of the heuristic, we compared the manual portfolio solution for a real-world instance against the solution generated by the algorithm for that same instance. The manual portfolio solution was built by expert managers and engineers at the company.

In order to assess the robustness of the procedure, we used the real-world instance provided by the company as a seed for generating similar instances, and randomly disturbed the seed instance data, in a controlled way and by small margins. The disturbance procedure applied the following modifications to the real instance data, where $d$ was a fixed disturbance factor:

Project costs: modified by $x \%$, with $x$ randomly chosen between $-d$ and $+d$.

Warning point risks: modified by $x \%$, with $x$ randomly chosen between $-d$ and $+d$.

Other parameters: not modified in the disturbed instances.

Using these modifications, we generated 10 instances for a 5\% disturbance factor. We decided to adopt the disturbance factor of $5 \%$ because, according to engineers, modifications in the overall cost beyond $5 \%$ in a year are unlikely in a traditional industry such as in the electrical power generation industry, whose assets do not change by a large amount yearly.

\subsection{Heuristic parameters}

Before running the robustness experiment, we performed some calibration in order to identify adequate values for some of the GRASP parameters.

During the construction phase, the procedure chooses between the HRSP and the SP lists with probabilities $\eta$ and $1-\eta$, respectively. In order to choose a proper value for $\eta$, we executed the construction procedure until it obtained 1000 feasible solutions for each $\eta$ value. Table 4 shows the best and average objective function values, the number of unfeasible solutions constructed until 1000 feasible solutions are reached, and the running time, for selected $\eta$ values. Using the experiment's results, we set $\eta=0.6$ since this yields very good values for both best and 
Table 4 - Probability of choosing HRSP list $(\eta)$, best and average solution values ( $\mathrm{OF}$, for objective function; in $10^{6}$ risk-month units), number of unfeasible solutions (Unfeasible), and running time values (Time) in seconds.

\begin{tabular}{|c|cc|c|c|}
\hline$\eta$ & Best OF & avg OF & Unfeasible & Time \\
\hline 0.20 & 0.57182 & 0.63542 & 397 & 116 \\
0.25 & 0.57155 & 0.64131 & 72 & 89 \\
0.30 & 0.56832 & 0.64124 & 41 & 87 \\
0.35 & 0.57327 & 0.64124 & 9 & 84 \\
0.40 & 0.57392 & 0.64086 & 4 & 84 \\
0.45 & 0.57244 & 0.64014 & 2 & 85 \\
0.50 & 0.57702 & 0.64172 & 4 & 86 \\
0.55 & 0.56897 & 0.64071 & 5 & 86 \\
0.60 & 0.56927 & 0.64042 & 6 & 84 \\
0.65 & 0.56780 & 0.64067 & 4 & 85 \\
0.70 & 0.57194 & 0.64060 & 3 & 86 \\
0.75 & 0.56850 & 0.63603 & 2 & 86 \\
0.80 & 0.57321 & 0.63434 & 0 & 87 \\
0.85 & 0.57562 & 0.63427 & 0 & 86 \\
0.90 & 0.56814 & 0.63477 & 0 & 87 \\
0.95 & 0.56832 & 0.63560 & 1 & 98 \\
1.00 & 0.57046 & 0.63316 & 0 & 115 \\
\hline
\end{tabular}

average solution values, as well as gives rise to few unfeasible solutions and shows good running time figures. This choice increases the odds that a high-risk controlling project will be scheduled earlier in the portfolio.

While the construction procedure was producing a new portfolio, the next project and month pair, $(p, m)$, to be inserted in the current partial solution was randomly chosen among the first $k$ best candidates in the sorted candidate list. We set $k$ equal to 5 , because this value was a good compromise between enforcing the randomized aspect of the heuristic while not loosing the greedy advantage of having the candidate list sorted.

We performed several experiments (results not shown) to decide on the best values for parameters $C$, the solution pool size, and $\beta$, the number of solutions to carry on to the search phase. Since the local search phase is the most time consuming computation performed by the heuristic, it proved to be an advantage to use $\beta<C$. The experiments took as input the real instance, as well as the disturbed instances described in Section 7.1. We finally decided on 200 for the pool capacity $C$ and set $\beta$ to be $10 \%$ of the capacity.

For the local search phase, we experimented with distinct values for the parameter $\Delta$, used in finding neighbor solutions. We found empirically that setting $\Delta$ to 8 covers a good amount of neighbor solutions without compromising the algorithm's performance.

Table 5 summarizes the parameter values used in the heuristics. 
Table 5 - GRASP parameters values.

\begin{tabular}{|c|c|l|}
\hline Param. & Vale & Description \\
\hline$\eta$ & 0.6 & probability of choosing next project from the HRSP list \\
$k$ & 5 & $k$ best candidates to choose from \\
$C$ & 200 & solution pool size \\
$\beta$ & 20 & number of solutions to carry on to the search phase \\
$\Delta$ & 8 & $2 \times \Delta$ is the size of a neighborhood \\
\hline
\end{tabular}

\subsection{Results and analysis}

The GRASP based heuristic was executed once for each input instance, and the best solution was retained. The experiments were performed in an Intel ${ }^{\circledR}$ i3 Dual Core ${ }^{\circledR}$ HT $1333 \mathrm{GHz}$ CPU, with $4 \mathrm{~Gb}$ of RAM, running the Ubuntu GNU/Linux distribution, version 12.04 LTS, with kernel 3.5.0. The algorithm was implemented using the GO 1.1.2 programming language for its enhanced performance and ease of coding. In order to improve the algorithm's performance, both the construction and local search procedures were implemented using GO parallel programming features.

The results are summarized in Table 6. The first column shows the disturbance factor (DF) and an instance identification. A DF of $0 \%$ refers to the real instance. The second and third columns are, respectively, the best and the average optimized objective function (OOF) values in risk-month units, where a risk-month unit is, of course, one month times one risk-unit. The next column gives the objective function value for the corresponding manual portfolios (IOF) in risk-month units. The next to last column informs the total running time (Time) for executing one run for each instance, in seconds, and the last column (Remaining Resource) gives the total remaining resource values (in $10^{9}$ monetary units) when the best portfolio found adopted as a solution for the PPS.

Besides the objective function value, we also obtained the total amount of remaining resources after each portfolio was constructed, in order to check the impact of the heuristic on resource consumption. Table 6 contains a summary of the total remaining resources (in $10^{9}$ monetary units) along all the $\mathrm{PH}$, and per input instance. The first line corresponds to the undisturbed instance, and each of the subsequent lines gives the result for the corresponding disturbed instance, as described in Section 7.1.

The results show that the heuristic consistently found solutions whose objective function values were around $47 \%$ better when compared to the corresponding values for the manual, undisturbed solutions. This behavior was repeated when the algorithm took as input any of the disturbed instances, attesting to its robustness when relatively small changes were applied to the real input instance.

Figure 2 shows some optimized (OOF) values statistics for two typical instances. The data is summarized in two boxplots: the first one presents statistics for the OOF values (in $10^{6}$ riskmonth units) obtained from the real instance (DF0). The second boxplot presents the OOF statistics for a $5 \%$ perturbed instance (DF5). 
Table 6 - Best, average, and input instance solution values (in $10^{6}$ risk-month units), and running time values (in seconds) for distinct input instances. The last column contains total remaining resource values (in $10^{9}$ monetary units) for the best solution.

\begin{tabular}{|c|cc|c|c|c|}
\hline DF - instance & min OOF & avg OOF & IOF & Time & Remaining Resource \\
\hline $0 \%$ & 0.54682 & 0.58865 & 0.99631 & 285.506 & 0.328 \\
$5 \%-\mathrm{a}$ & 0.51916 & 0.56113 & 0.99907 & 129.133 & 0.252 \\
$5 \%-\mathrm{b}$ & 0.52152 & 0.55443 & 0.97661 & 131.456 & 0.259 \\
$5 \%-\mathrm{c}$ & 0.51454 & 0.55913 & 0.98841 & 136.996 & 0.285 \\
$5 \%-\mathrm{d}$ & 0.54014 & 0.57090 & 0.98184 & 139.128 & 0.271 \\
$5 \%-\mathrm{e}$ & 0.52945 & 0.56467 & 0.97402 & 131.480 & 0.279 \\
$5 \%-\mathrm{f}$ & 0.51884 & 0.55234 & 0.98141 & 149.432 & 0.254 \\
$5 \%-\mathrm{g}$ & 0.53923 & 0.57053 & 0.99235 & 134.487 & 0.251 \\
$5 \%-\mathrm{h}$ & 0.54274 & 0.58001 & 0.99567 & 143.270 & 0.270 \\
$5 \%-\mathrm{i}$ & 0.51250 & 0.56150 & 0.97650 & 132.105 & 0.334 \\
$5 \%-\mathrm{j}$ & 0.52402 & 0.56475 & 0.98433 & 135.379 & 0.255 \\
\hline
\end{tabular}

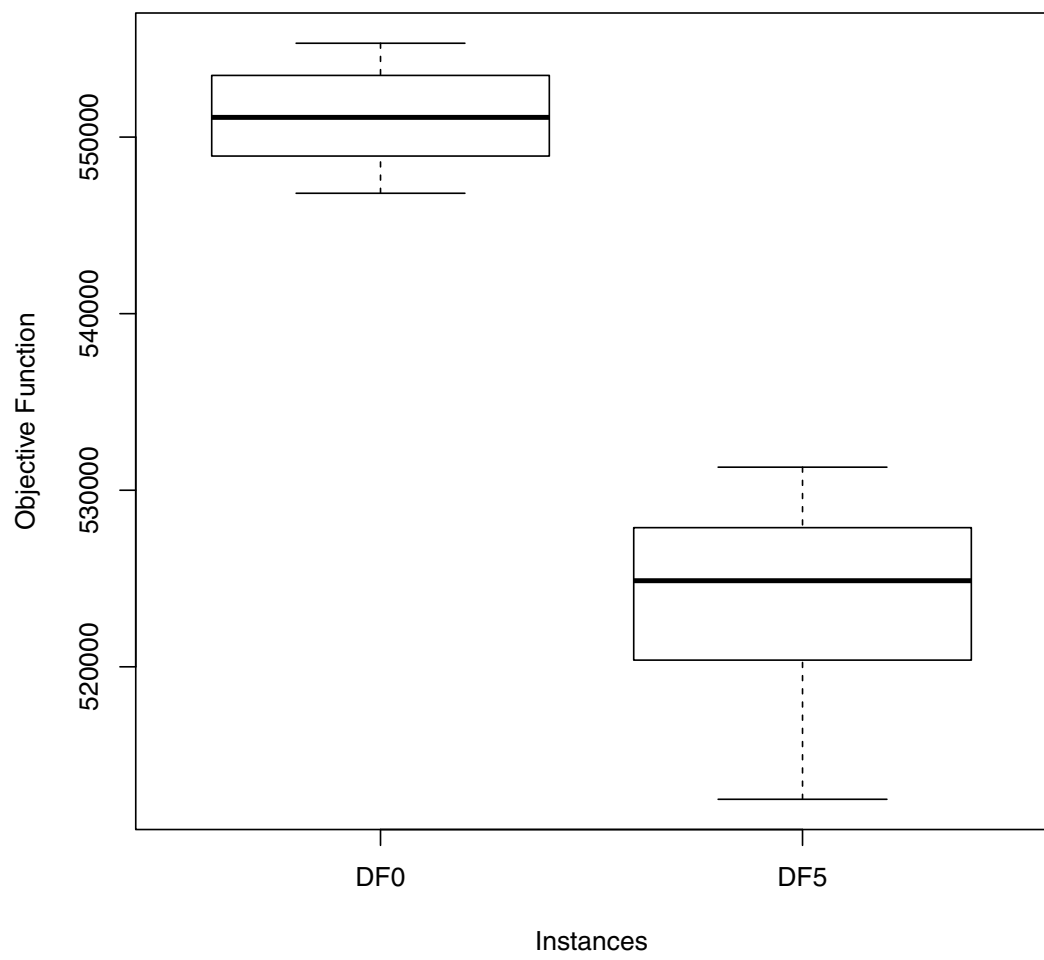

\begin{tabular}{ccccccc}
\hline Instance & Min & 1st Q & Median & Mean & 3rd Q & Max \\
\hline DF0 & 0.54682 & 0.56629 & 0.57926 & 0.58865 & 0.59529 & 0.70257 \\
\hline DF5 & 0.51250 & 0.53731 & 0.54994 & 0.56150 & 0.56828 & 0.70926 \\
\hline
\end{tabular}

Figure 2 - Best solution value statistics for the real instance and a perturbed instance. 


\section{CONCLUSIONS}

We modeled a project portfolio selection (PPS) problem derived from a large electrical power generation and distribution company, operating in Brazil. Besides traditional obstacles, such as resource constraints, the problem also involves complex features, such as elaborate exclusion dependencies among projects and an intricate relationship among projects that control warning points.

We proposed a variant of the GRASP meta-heuristic for solving this specific PPS problem. We modified the construction phase by using a greedy randomized procedure to construct feasible solutions that are stored in a pool of solutions, instead of the standard practice of constructing a single solution from a single candidate list. Firstly, the greedy procedure used two candidate lists to distinguish high-risk versus low-risk projects. Secondly, only a fraction of the best solutions in the pool are fed into the local search phase, which uses a best improvement strategy. Neighbor solutions used in the local search procedure are defined by changing a project's scheduled time by a small amount.

While designing the heuristics, we were not sure about which one of the two construction strategies, namely adaptive or non-adaptive, would provide better solutions. Therefore, we experimentally evaluated their performance and the quality of their solutions using a real-world input instance. It turned out that the non-adaptive strategy produced better solutions within a smaller running time, even when we adopted different adaptive benefit functions. In this particular experiment, the average value from all solutions produced by the non-adaptive strategy were nearer to the overall best solution values than any best solution produced by any of the adaptive procedures tested.

We performed experiments using real-world input instances in order to find appropriate parameters for the heuristics and to assess the algorithm robustness. The heuristics showed a significant improvement, up to around $47 \%$, over the manual solutions when treating instances of real data, as well as when taking disturbed data as input. We note that when creating these manual solutions, company experts were, likewise the optimization routine, mainly concerned with controlling the risks as soon as possible, so that comparing both solutions, at this stage, was meaningful and fair.

As for future work we can envisage the inclusion of new constraints concerning different categories of available resources, such as human resources, the concurrent use of multiple objective functions, and versions of the problem where projects can be made available, or be dismissed, in a dynamic fashion. That is, given an optimized portfolio $P$, after the company started executing the projects scheduled as indicated in $P$, some urgent new projects might arrive which would have to be inserted in $P$, maybe dropping other projects that did not yet started. It would be interesting to have a, maybe lighter, algorithm that could modify $P$ accordingly. In a similar fashion, projects in $P$ that did not yet started could be deemed as no longer necessary and, in this case, such projects could be dropped from $P$, maybe making room to accommodate in $P$ other projects that were left out originally because of resource restrictions. As another point, the heuristic could also include more sophisticated adaptive procedures in the construction phase, as well as improvements in the performance of the local search by adopting different neighborhood search strategies. 


\section{ACKNOWLEDGMENTS}

The authors would like to thank the company for their technical support and real data sets, and thank the National Agency for Electrical Energy (ANEEL) for their financial support (grant PD 0064-1021/2010).

\section{REFERENCES}

[1] Anandalingam G \& Olsson C. 1989. A multi-stage multi-attribute decision model for project selection. European Journal of Operational Research, 43(3): 271-283.

[2] ARCher N \& GHASEMZADeh F. 1998. A decision support system for project portfolio selection. International Journal of Technology Management, 16(1): 105-114.

[3] Archer N \& Ghasemzadeh F. 1999. An integrated framework for project portfolio selection. International Journal of Project Management, 17(4): 207-216.

[4] BAKER N \& FREELAND J. 1975. Recent advances in R\&D benefit measurement and project selection methods. Management Science, 21(10): 1164-1175.

[5] BenAiJa K \& KJIRI L. 2015. Project portfolio selection: Multi-criteria analysis and interactions between projects. CoRR, abs/1503.05366.

[6] Campbell J \& Campbell R. 2001. Analyzing and managing risky investments. Corporate Publishing International, Grosvenor Business Tower, Office 804, Dubai 13700, United Arab Emirates. 1st edition, ISBN-13: 978-0970960702.

[7] Carazo A, Gómez T, Molina J, Hernández-Díaz A, Guerrero F \& Caballero R. 2010. Solving a comprehensive model for multiobjective project portfolio selection. Computers \& Operations Research, 37(4): 630-639.

[8] Chiam S, Tan K \& Al Mamum A. 2008. Evolutionary multi-objective portfolio optimization in practical context. International Journal of Automation and Computing, 5(1): 67-80.

[9] Doerner K, Gutjahr W, Hartl R, Strauss C \& Stummer C. 2004. Pareto ant colony optimization: A metaheuristic approach to multiobjective portfolio selection. Annals of Operations Research, 131(1): 79-99.

[10] FeStA P \& RESENDE M. 2011. GRASP: basic components and enhancements. Telecommunication Systems, 46(3): 253-271.

[11] Fox G, BAKER N \& BRYANT J. 1984. Economic models for R and D project selection in the presence of project interactions. Management Science, 30(7): 890-902.

[12] Gutjahr W, Katzensteiner S, Reiter P, Stummer C \& Denk M. 2010. Multi-objective decision analysis for competence-oriented project portfolio selection. European Journal of Operational Research, 205(3): 670-679.

[13] Henriksen A \& Traynor A. 1999. A practical R\&D project-selection scoring tool. Engineering Management, IEEE Transactions on, 46(2): 158-170.

[14] Herroelen W \& Leus R. 2005. Project scheduling under uncertainty: Survey and research potentials. European Journal of Operational Research, 165(2): 289-306. 
[15] ISO 2009a. ISO GUIDE 31000:2009 “Risk management-Principles and guidelines”. International Standards Organization, Chemin de Blandonnet 8, CP 401, 1214 Vernier, Geneva, Switzerland. Prepared by IEC Technical Committee 56 - 'Dependability', and the ISO Technical Management Board's Risk Management Working Group.

[16] ISO 2009b. ISO GUIDE 73:2009 “Risk Management-Vocabulary”. International Standards Organization, Chemin de Blandonnet 8, CP 401, 1214 Vernier, Geneva, Switzerland. Prepared by the ISO Technical Management Board Working Group on Risk Management.

[17] Kienzle F, Koeppel G, Stricker P \& Andersson G. 2007. Efficient electricity production portfolios taking into account physical boundaries. In Proceedings of the 27th USAEE/IAEE North American Conference.

[18] LEE J \& KIM S. 2000. Using analytic network process and goal programming for interdependent information system project selection. Computers \& Operations Research, 27(4): 367-382.

[19] LIESIÖ J, MiLd P \& SALO A. 2008. Robust portfolio modeling with incomplete cost information and project interdependencies. European Journal of Operational Research, 190(3): 679-695.

[20] LIU M \& WU F. 2007. Portfolio optimization in electricity markets. Electric Power Systems Research, 77: 1000-1009.

[21] Martinsuo M \& Lehtonen P. 2007. Role of single-project management in achieving portfolio management efficiency. International Journal of Project Management, 25(1): 56-65.

[22] MeAdE L \& PREsley A. 2002. R\&D project selection using the analytic network process. Engineering Management, IEEE Transactions on, 49(1): 59-66.

[23] Mira C, Feijão P, Souza M, Moura A, Meidanis J, Lima G, Bossolan R \& Freitas I. 2012. A GRASP-based heuristic for the Project Portfolio Selection Problem. In The 15th IEEE International Conference on Computational Science and Engineering (IEEE CSE2012), volume 1, pages 36-41, Paphos, Cyprus. Sponsored by IEEE. ISBN 978-1-4673-5165-2.

[24] Mohanty R, Agarwal R, Choudhury A \& TiWari M. 2005. A fuzzy ANPbased approach to R\&D project selection: a case study. International Journal of Production Research, 43(24): 5199_ 5216.

[25] Rabbani M, Aramoon Bajestani M \& Baharian Khoshkhou G. 2010. A multiobjective particle swarm optimization for project selection problem. Expert Systems with Applications, 37(1): $315-321$.

[26] RESENDE MGC. 2009. Greedy Randomized Adaptive Search Procedures. In Encyclopedia of Optimization, pages 1460-1469. Springer.

[27] Shou Y, Xiang W, Li Y \& Yao W. 2014. A Multiagent Evolutionary Algorithm for the ResourceConstrained Project Portfolio Selection and Scheduling Problem. Mathematical Problems in Engineering, 2014(302684): 1-9.

[28] Szolgayová J. 2010. CVaR Portfolio Models for Electricity Generating Capacities. PhD thesis. 